\title{
New readout system for the KOTO CsI calorimeter upgrade
}

\section{Tomoo Mari*}

Osaka University, Department of Physics

E-mail: mariechamp.hep.sci.osaka-u.ac.jp

The J-PARC KOTO experiment searches for the rare kaon decay $K_{L} \rightarrow \pi^{0} v \bar{v}$. The CsI calorimeter is to measure two photons from $\pi^{0}$ in the final state, and the CsI crystals are read out from the rear end with photomultiplier tubes (PMTs). Background neutrons can be discriminated from photons by comparing the shower depth in the calorimeter. To measure the depth through the arrival-time difference between the front and rear ends, 4096 multi pixel photon counters (MPPCs) will be put on the front surface of the calorimeter. This new readout system using MPPCs is reported in this paper.

ICHEP 2018, International Conference on High Energy Physics

4-11 July 2018

Seoul, Korea

${ }^{*}$ Speaker. 


\section{Neutron-induced background in the $K_{L} \rightarrow \pi^{0} v \bar{v}$ experiment}

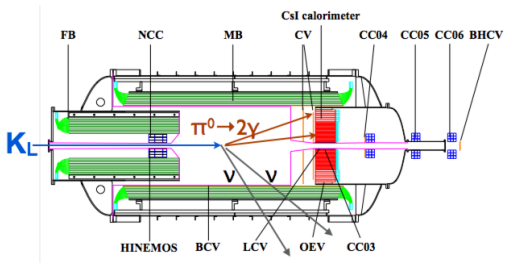

(a)

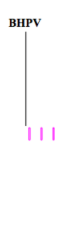

Figure 1: (a) KOTO detector; (b) beam halo neutron background.

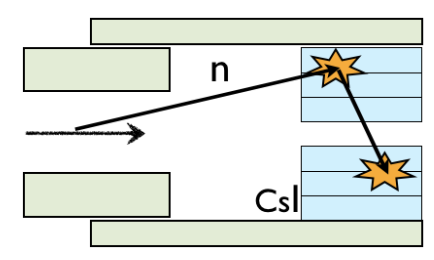

(b)

The aim of the J-PARC KOTO experiment is to search for new physics beyond the Standard Model (SM) by measuring the rare kaon decay $K_{L} \rightarrow \pi^{0} v \bar{v}$. This decay is highly suppressed in the SM, and its branching ratio is predicted to be $(3.0 \pm 0.3) \times 10^{-11}$. The KOTO detector consists of a CsI calorimeter and hermetic veto counters (Figure $\mathrm{W(a))}$ ). The calorimeter consists of 2716 undoped CsI crystals, which are read out from their rear end with photomultiplier tubes (PMTs). The largest background source in the first physics run was so called the "halo neutron background"[四, in which neutrons scattered at the beam collimators hit CsI and make a hadronic shower, and a neutron in the shower propagates and produces the second shower (Figure $\mathbb{W}(\mathrm{b})$ ). To reach the standard model sensitivity, this background should be reduced by another factor of 10 .

\section{Both-end readout system for the CsI calorimeter}

Photons from $\pi^{0}$ decay produce showers in the upstream part of the calorimeter due to the short radiation length of CsI $(\sim 2 \mathrm{~cm})$. The second shower in the neutron background events tends to be located in the downstream part because the interaction length of CsI $(\sim 40 \mathrm{~cm})$ is comparable to the length of the crystals $(50 \mathrm{~cm})$. Thus, the depth of the showers is a good discriminator of the signals from the backgrounds. In 2018, we will put 4096 multi pixel photon counters (MPPCs) on the front-end surface of the crystals. One MPPC will be glued on every $2.5 \mathrm{~cm}$-squared region.

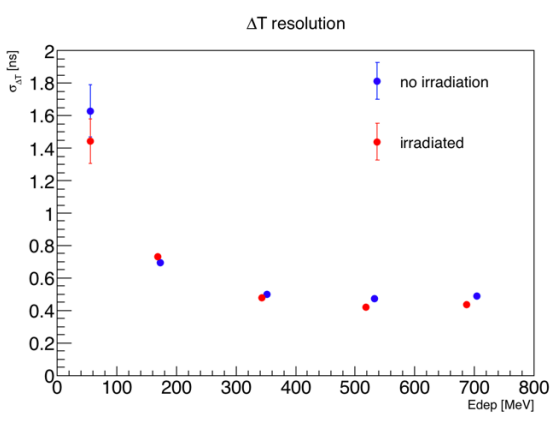

Figure 2: Resolution of timing difference between MPPCs read out with summing amplifier and a PMT. The shower depth is measured through the arrival-time difference of scintillation lights between the MPPC and the PMT. To reduce the number of readout channels, four MPPCs are connected together in front of the CsI surface and the four outputs are summed with amplifier circuits. We performed a beam test with $200-800 \mathrm{MeV}$ positrons to validate this new readout system at Research Center for Electron Photon Science (ELPH) of Tohoku University in December 2017. As a result, the timing resolution of the readout system was comparable with that of the individual crystal readout, and MPPCs irradiated by neutrons to the level expected for $3 \times 10^{7} \mathrm{~s}$ with $100 \mathrm{~kW}$ beam power worked as well as non-irradiated ones (Figure $\mathbb{2}$ ).

\section{References}

[1] J.K.Ahn, et. al., PTEP, (2017) $021 \mathrm{C} 01$. 